\title{
Morphology Controlled Low-dimensional Single-crystalline SnSe2-graphene Hybrid for near IR Photodetection
}

Rajeev Rai, Saurav Islam, Ahin Roy, Garvesh Agrawal, Abhishek Kumar Singh, Arinadm Ghosh and N Ravishankar

Indian Institute of Science, Bangalore, Bangalore, Karnataka, India

With the ingenious discovery and development in the synthesis of ultrathin graphene, layered metal dichalcogenides (LMCs) $\left(\mathrm{MX}_{2}\right)$ have gained enormous attention in catalysis, ${ }^{1}$ electronics, ${ }^{2}$ optoelectronics ${ }^{3}$ and sensing. ${ }^{4}$ LMCs shows diverse range of properties which can be further tuned depending on the shape and size. Crystallography, LMCs have metal atom sandwiched between two layers of chalcogen as X-M-X bonded by covalent bonds and each X-M-X layer are weakly bonded by van der Waals forces.

$\mathrm{SnSe}_{2}$ being a member of LMC has layered structure of $\mathrm{CdI}_{2}$ type with $\mathrm{Sn}$ atom sandwiched between two layers of Se and has bulk optical band gap of $1 \mathrm{eV}$. This property can be utilized for near IR range photodetection. However, LMDs suffer from low mobility or localized nature of electron which can be overcome by making a binary hybrid device with graphene and thus, can provide a very high gain, leading to an ultra-sensitive photo-detector. ${ }^{5}$ For the better the interface and enhanced charge transfer a large flat interface between LMCs and graphene is needed. Therefore, stringent control over the morphology is needed in order to apply them for targeted applications.

Herein, $\mathrm{SnSe}_{2}$ has been synthesized by scalable one step solvothermal method in two different morphology viz. nanosheet and overgrown nanoflowers under two different conditions. In presence of only oleic acid as solvent, overgrown flower shaped $\mathrm{SnSe}_{2}$ forms while the use of excess ethanol along with oleic acid as solvent leads to the formation of $\{0001\}$ faceted nanosheets. Morphology and microstructural investigation of as-synthesized $\mathrm{SnSe}_{2}$ have been performed using scanning electron microscope and transmission electron microscope.

Figure 1 shows the microstructure of two different morphology of SnSe2. Figure 1(a) shows the assynthesized large flat $\mathrm{SnSe}_{2}$ nanosheets in presence of excess ethanol. Corresponding diffraction pattern of the sheet along [0001] is shown in figure 1(b). The hexagonally symmetric spots show the single crystalline nature of the nanosheet. High-resolution TEM micrograph of nanosheet has been shown in figure 1(c). In presence of only oleic acid, stacked overgrown $\mathrm{SnSe}_{2}$ flowers forms as shown in figure 1(d). However, Individual sheets of the flowers are single crystalline which can be seen from the SAED pattern as shown in the figure 1(e). Corresponding HRTEM micrograph is also shown in figure 1(f) showing the (10T0) lattice planes. STEM HAADF images of nanosheets are shown in figure 2, showing the hexagonal arrangement of Se atoms around Sn atom. 10 STEM micrograph of low dwell time have been acquired and averaged through rigid and non-rigid registration. ${ }^{6}$

The effect of ethanol and acid during the growth of $\mathrm{SnSe}_{2}$ was investigated using ab-inito DFT simulation, which reveals a stronger binding of ethanol as compared to acid group on (10T0) and thus inhibit the overgrowth of $\mathrm{SnSe}_{2}$ when present in the reaction medium and rationalizes our synthesis. 
A simple drop-cast based method was followed to fabricate graphene-SnSe2 nanosheets based binary hybrid device for near Infrared Photodetection. Fabricated device shows a transparent and trap free interface which is imperative for efficient performance of the device. Fabricated device shows the optical response in the IR regime $(920 \mathrm{~nm}, 1060 \mathrm{~nm}, 1300 \mathrm{~nm})$ and specific detectivity of device was found to be $5 \times 10^{10}$ jones, comparable to that of state-of-the-art photodetectors.

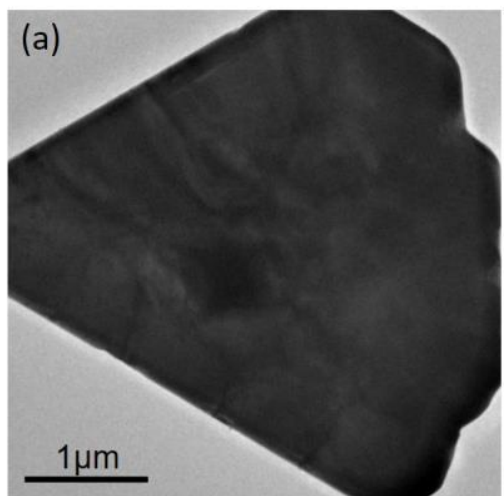

(d)

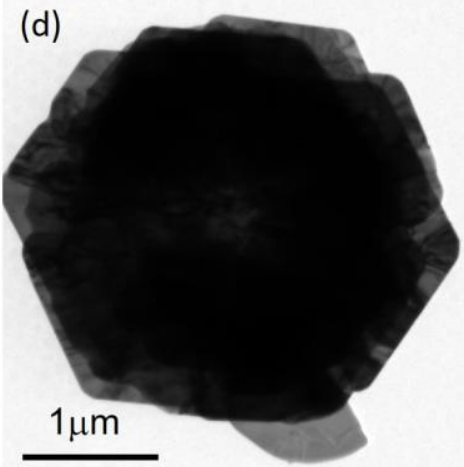

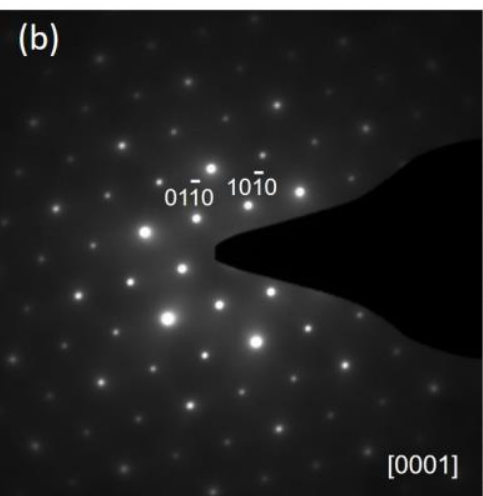

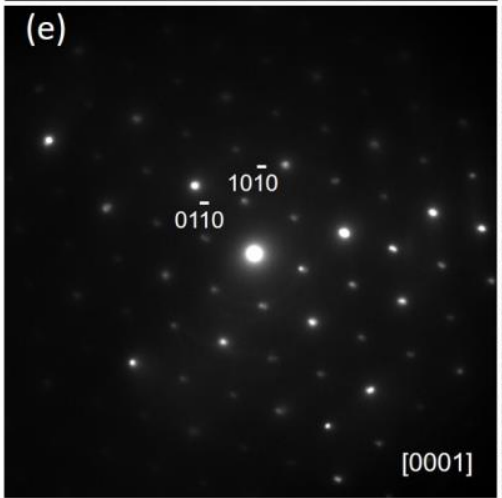

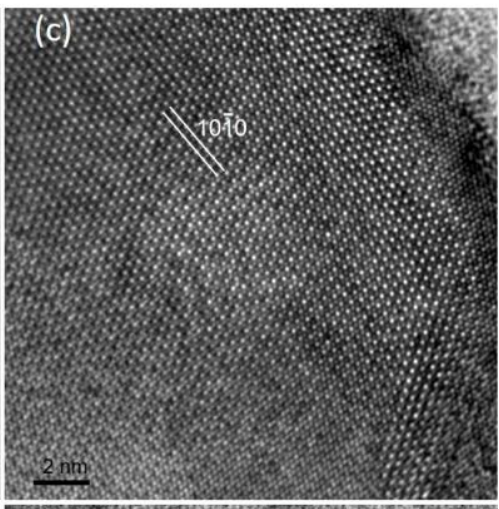

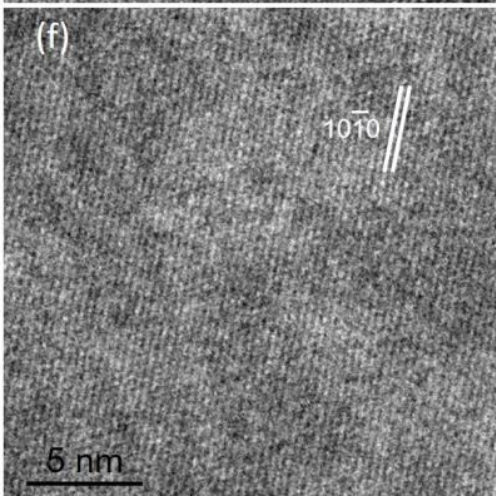

Figure 1. Bright-field TEM micrograph, selected area diffraction pattern showing the single crystalline nature of the individual flakes and high-resolution TEM micrograph of as-synthesized nanosheets $(a, b, c)$ and nanoflowers (d, e and f) respectively.
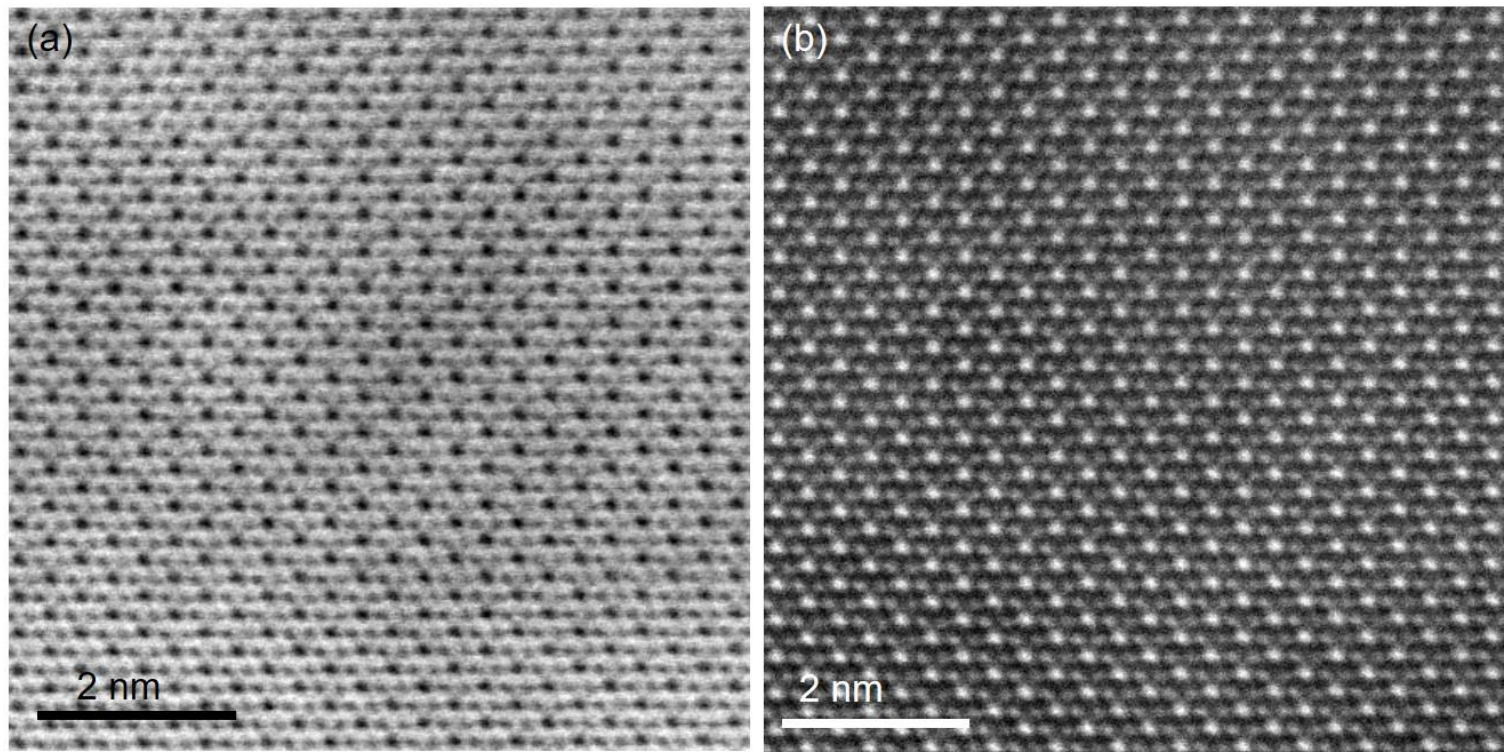
Figure 2. 10 frames average through stacked rigid and non-rigid aligned STEM micrograph, acquired at $300 \mathrm{kV}$ (a) bright-field; (b) HAADF micrograph showing the hexagonal arrangement of Se atoms around Sn atoms.

References

1. Li, J. Xiao, Q. Fu and X. Bao, Proc. Natl. Acad. Sci. U. S. A., 2017, 114, $5930-5934$.

2. Fiori, F. Bonaccorso, G. Iannaccone, T. Palacios, D. Neumaier, A. Seabaugh, S. K. Banerjee and L. Colombo, Nat. Nanotechnol., 2014, 9, $768-779$.

3. Huang, K. Xu, Z. Wang, T. A. Shifa, Q. Wang, F. Wang, C. Jiang and J. He, Nanoscale, 2015, 7, 17375 -17380 .

4. Anichini, W. Czepa, D. Pakulski, A. Aliprandi, A. Ciesielski and P. Samorì, Chem. Soc. Rev., 2018, 47, $4860-4908$.

5. Pradhan, A. Roy, S. Tripathi, A. Som, D. Sarkar, J. K. Mishra, K. Roy, T. Pradeep, N. Ravishankar and A. Ghosh, Nanoscale, 2017, 9, $9284-9290$.

6. Jones, H. Yang, T. J. Pennycook, M. S. J. Marshall, S. V. Aert, N. D. Browning, M. R. Castell \& P. D. Nellist, Adv Struct Chem Imag, 2015, 1(8). 